\title{
Re: Survival and Tolerability of Transarterial \\ Chemoembolization in Greater Versus less than 70 Years of Age \\ Patients with Unresectable Hepatocellular Carcinoma-A Propensity Analysis
}

\author{
Zhaonan $\mathrm{Li}^{1} \cdot$ Xinwei $\operatorname{Han}^{2}$ (1)
}

Received: 24 October 2020/Accepted: 31 October 2020/Published online: 17 November 2020

(C) Springer Science+Business Media, LLC, part of Springer Nature and the Cardiovascular and Interventional Radiological Society of Europe (CIRSE) 2020

To the editor,

We read with great interest the article by Dr. Mosconi et al. [1]. This retrospective study investigates the safety and efficacy of conventional transarterial chemoembolization (TACE) in elderly HCC patients compared with younger adults, and used propensity score matching (PSM) analysis on two different treatment methods, and pointed out that confirms that TACE is well tolerated and effective in patients aged 70 years or more with unresectable $\mathrm{HCC}$ as it is for their younger counterparts ( $<70$ years). Moreover, the article further explains liver-related mortality was not associated with age $\geq 70$ years and primarily predicted by tumor multifocality, Child-Pugh class B and an increased alpha-fetoprotein value $(>31 \mathrm{ng} / \mathrm{ml})$.

First, we congratulate the authors on the perfect match in this study. Twelve variables were included in the PSM analysis. However, the precision of the PSM analysis was not mentioned. Second, gross pathological type, Child-Pugh class, and a-fetoprotein level were all regarded as matching variables in the whole cohort. While it is noteworthy that, in the subgroup analysis for patients with solitary type and for patients with Child-Pugh B, patients were selected from the cohort after the PSM analysis. Patients should have been selected from the whole cohort according to the subgroup

Xinwei Han

13592583911@163.com

1 Department of Interventional Radiology, The First Affiliated Hospital of Zhengzhou University, Zhengzhou 450052, China

2 Department of Interventional Radiology, First Affiliated Hospital of Zhengzhou University, No. 1 Jianshe East Road, Zhengzhou City 450000, Henan Province, China criteria before the PSM analysis. The variables of gross pathological type, Child-Pugh class, and a-fetoprotein level should have been excluded when matching. The results obtained by this method are more convincing. Third, middleaged or elderly people usually have other comorbidities, which may be an important factor affecting the survival and prognosis of patients [2, 3], but there is no relevant involvement in the patient enrollment criteria of the article, which may lead to biased results.

In conclusion, we appreciate the authors' efforts in exploration of the treatment with elderly patients. However, we suggest that appropriate modification and improvement in statistical analysis would further confirm and greatly solidify the conclusions of the study.

\section{Compliance with Ethical Standards}

Conflict of interest The authors declare that they have no conflict of interest.

\section{References}

1. Mosconi C, Gramenzi A, Biselli M, et al. Survival and tolerability of transarterial chemoembolization in greater versus less than 70 years of age patients with unresectable Hepatocellular Carcinoma: a propensity score analysis. Cardiovasc Interv Radiol. 2020;43(7):1015-24.

2. Sun J, Zhou G, Zhang Y, et al. Comprehensive analysis of common safety profiles and their predictive factors in 520 records of liver cancer patients treated by drug-eluting beads transarterial chemoembolization. Medicine (Baltimore). 2018;97(26):e11131.

3. Hung AK, Guy J. Hepatocellular carcinoma in the elderly: metaanalysis and systematic literature review. World J Gastroenterol. 2015;21(42):12197-210.

Publisher's Note Springer Nature remains neutral with regard to jurisdictional claims in published maps and institutional affiliations. 